\title{
LOCAL CONCENTRATION OF SLOWLY VARYING WAVE AND CURRENT FIELDS AROUND THE ABRUPTLY CHANGING BOTTOM SLOPES ALONG THE SHORE
}

\author{
Yoshimitsu Tajima $^{1}$ and Shinji Sato ${ }^{1}$
}

\begin{abstract}
This study aims to investigate physical mechanisms of locally concentrated nearshore hydrodynamic forces under interactive fields of waves and currents on abruptly changing coastal bathymetry. Laboratory experiments were first performed to represent such phenomena and a newly developed monitoring system based on image processing techniques successfully captured the local concentrations of wave fields as quantitative high-resolution data sets. Numerical experiments were then performed to investigate the physical mechanisms of the observed features. It was found that, among the other various possible factors, slowly varying wave-induced nearshore currents have one of the most significant impacts on local concentrations of waves around the abruptly changing bathymetry.
\end{abstract}

Keywords: wave-current interaction, wave breaking, wave envelope, nearshore current, long wave

\section{INTRODUCTION}

The typhoon No.9, T0709, hit the Pacific Coast of Japan in September 2007 and caused severe damages along the coasts. Seisho coast, located in the middle of Sagami bay, was one of coasts which suffered most significant damages due to T0709 (Tajima and Sato, 2009). Figure 1 shows the bathymetry around the most damaged area of the Seisho coast and alongshore distributions of inundation heights measured after the storm. While Seisho coast has long and straight coast line, as seen in the figure, damaged area was concentrated behind Oiso-Spur, where the area with relatively shallow water depth of $h<100 \mathrm{~m}$ is convexly extended toward the sea. Measured inundation heights show somewhat periodic undulation along the coast and the locations of the highest peak of the inundation heights surely correspond to that of most damaged area where a part of highway along the coast was collapsed. According to Sato et al. (1998), the typhoon No.20, in 1997 (T9720) also hit near the Seisho coast and caused locally concentrated coastal damages while the most damaged area due to T9720 was about $6 \mathrm{~km}$ west from that of T0709. Similar locally concentrated coastal damages can be observed in Japan and these damages seem to have certain similarities such as: (i) the coast has abruptly changing bathymetry along the shore; and (ii) slowly varying long wave components were also significant during the storm.

The goal of this study is to investigate physical mechanisms of such features of locally concentrating hydrodynamic forces that eventually cause severe local damages along the coast. In this study, laboratory experiment is first carried out to represent such locally concentrating wave and current fields near the abruptly changing bathymetry under waves with low frequency wave

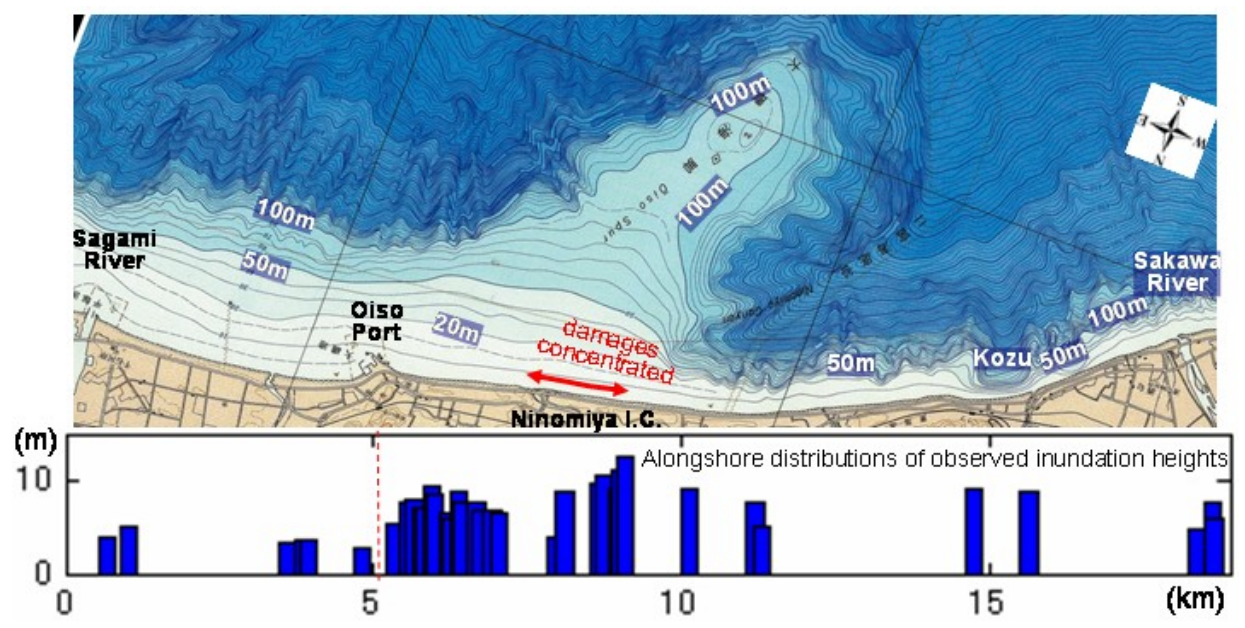

Figure 1 Measured inundation heights due to T0709 along the Seisho coast.

\footnotetext{
${ }^{1}$ Department of Civil Engineering, The University of Tokyo, Hongo 7-3-1, Bunkyo-ku, Tokyo 113-8656, Japan
} 
components. Image-based measuring system is then newly developed to capture the hydrodynamic characteristics in a wider area with higher resolution both in time and space domains. Finally, numerical analysis is performed to further investigate the physical mechanisms of the observed features of locally concentrated hydrodynamic forces.

\section{LABORATORY EXPERIMENT}

Laboratory experiment was first performed to explore the physical mechanisms of locally concentrating hydrodynamic force due to various factors such as abruptly changing bathymetry and interactions among wind waves, currents and low-frequency wave components.

\section{Experimental Setups}

Figure 2 shows plane view of the experimental setups. As seen in the figure, the original bed of the basin consists of a plane panel with constant water depth near the wave generator and two different shore-parallel inclined planes with cross-shore slopes of 1:10 and 1:20, respectively. In the alongshore half of the basin, stainless attachments were placed so as to create gently sloping 1:40 plane on top of the 1:20 sloping plane. In this manner, the basin somewhat represents the bathymetry at Seisho coast where relatively straight coasts with different cross-shore slopes merge each other at the Oiso Spur (Figure 1). Boundary between panels with different slopes, 1:20 and 1:40, were linearly connected by plane panels with an alongshore slope of 1:10. As seen in Figure 2, furthermore, white light source of rectangular panel was imbedded in the 1:40 sloping panel near the boundary of two different bottom slopes. The water was colored in blue using artificial coloring, food blue No.2 CI 73015 indigo carmine. Video camera was then installed above the light source and captured the image of the light penetrating through the colored water. Captured pixel data was then transferred to the brightness and compared to the local water depth. Near the shoreline, vertical sea wall was placed along the straight $2 \mathrm{~cm}$-deep contour lines of still water depth. The sea wall was colored in yellow and the other four video cameras, illustrated in Figure 2, were installed to capture the surface water fluctuations on the sea wall. A part of the seawall, ranging within $-40(\mathrm{~cm})<y<-20(\mathrm{~cm})$ as shown in the Figure 2, was replaced by a transparent acrylic panel and, behind the transparent seawall, a plane mirror plate was placed. Green laser sheet was then introduced from the top of the mirror and, by adjusting the angle of the mirror plate, the reflected laser sheet was introduced through the transparent panel to the water and radiated thin layer parallel to the sloping floor and at height of $5 \mathrm{~mm}$ above the bottom bed. This system enabled us to introduce the thin laser layer from the fixed boundary to the water but not from the fluctuating surface water boundary without disturbing the laser layer due to refraction at the fluctuating air-water boundary. Aluminum powder was then put into the water and the illuminated powder in the thin green laser layer was captured by high-speed camera with frame rate of $100 \mathrm{fps}$. Particle tracking velocimetry, PTV, was then applied to the obtained images to yield instantaneous horizontal velocity components along the green laser layer.

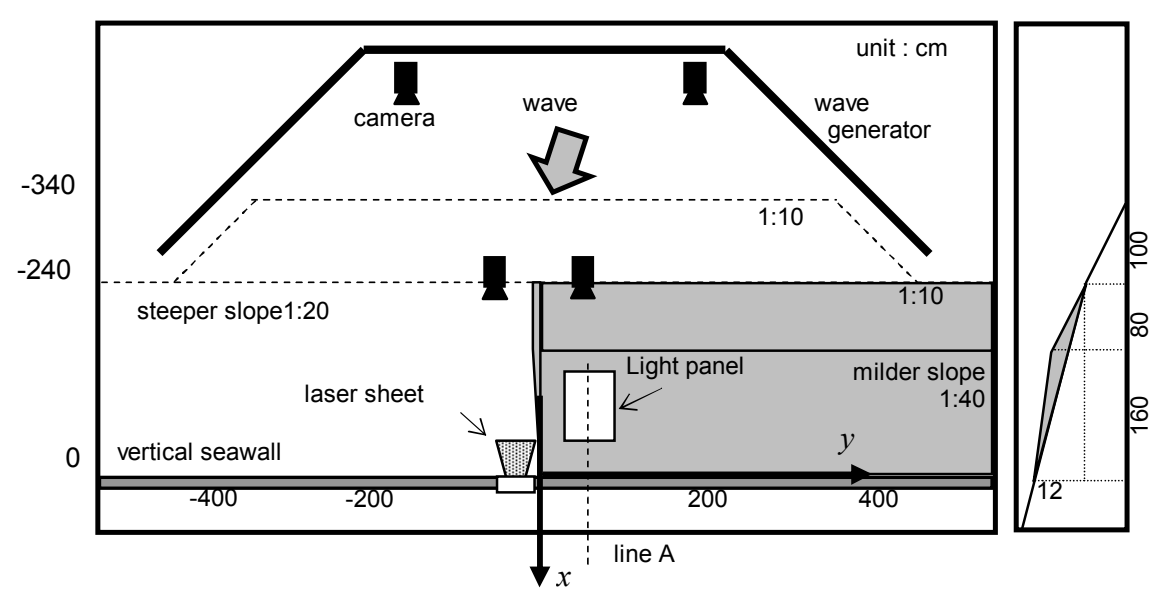

Figure 2 Layout of the experimental setup 
In this basin, this study introduced either monochromatic sinusoidal wave or bichromatic waves, i.e., combinations of two monochromatic sinusoidal waves whose wave frequencies are slightly offset from each other. In this manner, bicromatic wave develops slowly varying wave envelope which induces low-frequency band waves as well as free long waves especially after the wave breaking in the surf zone. Wave period and height of the regular monochromatic wave were set to be $T=1 \mathrm{~s}$ and $H_{0}=2.8 \mathrm{~cm}$, respectively. Similarly, wave properties of combining two wave components were respectively set to be $H_{0}=2 \mathrm{~cm}$ and $T=1.111 \mathrm{~s}$ or $0.909 \mathrm{~s}$. These two wave heights were selected so that averaged energy of the combined waves becomes equivalent to the monochromatic wave and the timeperiod of the envelope becomes $5 \mathrm{~s}$. At the wave generator with uniform water depth $25 \mathrm{~cm}$, these waves were introduced with incident angle of 10 degrees. The wave generator was controlled so as to absorb the reflected wave components whose properties were estimated from the measured surface water fluctuations in front of the wave generator.

\section{Image-Based Data Acquisition System}

Based on the succeeding still digital images captured by all these six cameras including the highspeed camera, we developed a data acquisition system which yields widely covering and high resolution data sets of current and surface water fluctuations.

Obtained images were first rectified to quantify the distance and directions in the image based on the actual xyz coordinate system. Based on seven bench-mark points, which determine geometric relationships between actual xyz coordinates and image pixel coordinates, rectification parameters, such as camera locations, angles, focal length and lens distortion, were estimated so that the obtained parameters yield the minimum root-mean-square errors of estimated pixel coordinates at seven benchmark points. This study also applied weight function proposed by Tajima et al.(2009) so that the relative importance of the errors at each bench mark points based on the xyz coordinates but not image pixel coordinates become equivalent to each other. The other detailed procedures, validations, and primary initial results of the measured system are summarized in the following respective three measuring items.

Surface Water Fluctuations along the Seawall Based on the succeeding still images recorded by four cameras which captured the seawall from seaside toward the shore, instantaneous surface water fluctuations along the sea wall were estimated. The horizontal range of the image captured in each camera was about $1 \mathrm{~m}$ along the sea wall and frame rate was set to be $30 \mathrm{fps}$.

Based on RGB-values in each pixel, the surface water boundary on the sea wall was detected by a following parameter,

$$
S_{1}=R+G-B
$$

Since yellow is represented by larger values of both $R$ and $G$ and blue, on the other hand, is expressed by larger $B$ and smaller $R$ and $G$, this parameter, $S_{1}$, gives a larger value on the sea wall and smaller value on the blue water. Figure 3(a) shows a snap-shot image of the sea wall when the broken wave was splashing on the wall. In this image, pixel data were replaced by the parameters $S_{1}$. Figure 3(b) shows the downward vertical distributions of $S_{l}$ along the line specified in the Figure 3(a). As seen in these figures, the parameter $S_{1}$ abruptly decreases on the splashing water even though the water layer is relatively thin and its color is not as dark as the one on the still deeper water. Since $S_{1}$ is nearly constant on the yellow sea wall, this study determined the surface water boundary condition of $S_{I}$ by a single critical value, $S_{I}=290$, which is very close to the value on the yellow seawall. Starting from the arbitrary points on the seawall, the system search the pixel location in the vertical downward direction where $S_{1}$ first goes below this critical value and the detected pixel coordinates were then transferred to the xyz-coordinates on the sea wall based on the obtained rectification parameters in advance. Estimated averaged error of the rectified coordinates was less than one pixel and the averaged pixel size in the xyz coordinate system on the sea wall was around $1.6 \mathrm{~mm}$. The possible error of this surface water detection system should therefore be of the same order.

Figure 4(a) compares the time-varying surface water fluctuations estimated by the present imagebased system and the ones measured by wave gauge. As seen in the figure, the estimated surface water profiles agree well with the ones measured by the wave gauge.

Surface Water Fluctuations on the Rectangular Light Panel Horizontal distributions of instantaneous surface water fluctuations was estimated on the rectangular light panel embedded in the 
1:40 sloping bottom based on the succeeding still images. Frame rate of the video image was set to be $60 \mathrm{fps}$ and all the light except the embedded light source was turned off so that the recorded images only capture the brightness of the bottom light source penetrating through the colored water column. Using the image taken on the still water condition, known water depth increasing in the offshore direction was compared with the local RGB values at each pixel. Through various comparisons, it was found that the parameter, $S_{2}=R+G$ showed the best correlations with the water depth. Relationship between this parameter, $S_{2}$, and the local water depth, $h$, was then fitted in the following simple exponential curve,

$$
S_{2}=\alpha \exp (-\beta h)
$$

with $\alpha$ and $\beta$, fitting coefficients which should be determined through the obtained data sets. Even on the same water depth along the shore-parallel contour line, the parameter $S_{2}$ was found to vary with the alongshore locations. This study therefore separately determined the optimum values of $\alpha$ and $\beta$ along each cross-shore line at different alongshore location. Local water depth was then inversely estimated through equation (2) with local $S_{2}$ and optimum sets of $\alpha$ and $\beta$ on the entire light source. The maximum error of estimated water depth was $2.5 \mathrm{~mm}$.

Figure 4(b) compares the surface water profile at the offshore corner of the light panel estimated by the present image-based measuring system and the one measured by the wave gauge. As seen in the

(a)
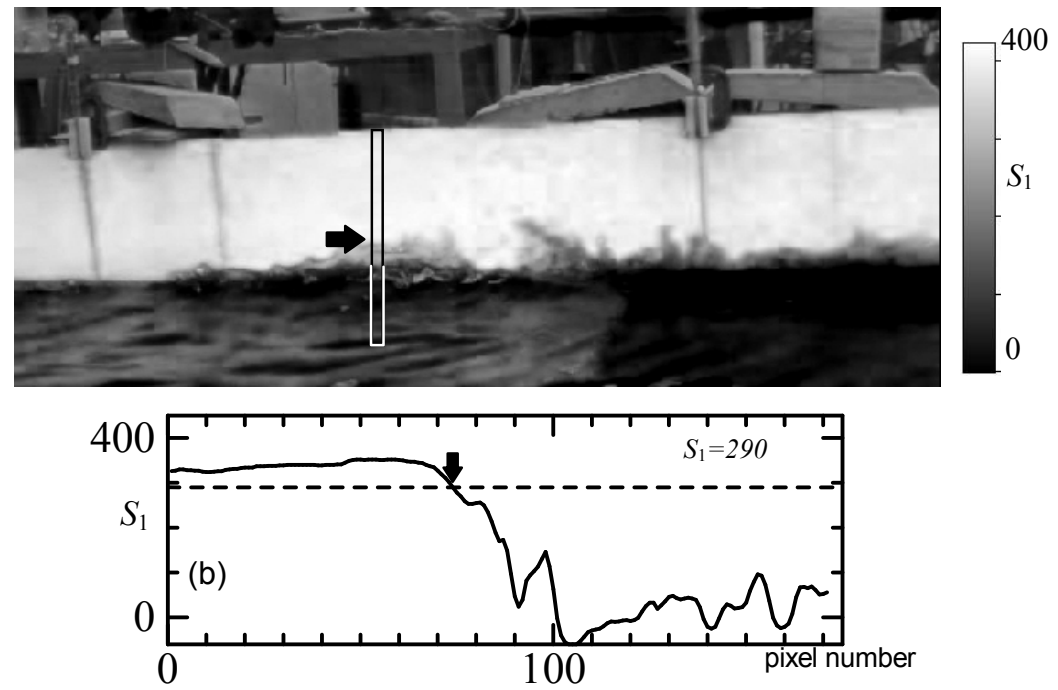

Figure 3 (a) Recorded image with pixel values replaced by $S_{1}$ and (b) vertical distributions of $S_{1}$ along the line specified in (a)
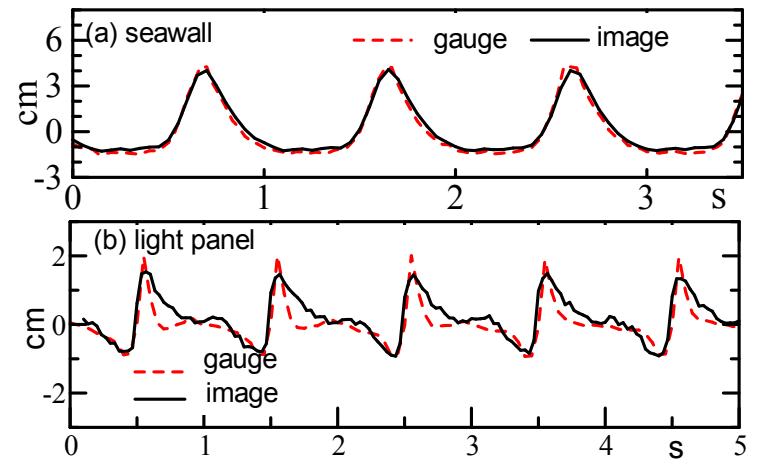

Figure 4 Comparisons of surface water fluctuations measured by a wave gauge and by the present imagebased measuring system at (a) seawall and (b) offshore corner of the light panel 
figure, the image-based system tends to estimate milder wave crest but is able to capture overall surface water profile within acceptable errors.

Horizontal Current Field Succeeding still images taken by a high speed camera in front of the seawall were finally applied for estimation of the horizontal velocity fields in the nearshore area. Aluminum powder illuminated by green laser layer was used as tracer and directly estimate the instantaneous and local horizontal velocity by taking time derivative of the obtained tracks of the tracers. By introducing the green laser outside of the seawall, a rigid transparent panel, laser was not disturbed by fluctuating surface water boundary and successfully illuminate a fixed thin layer about $5 \mathrm{~mm}$ above the bottom bed. To remove noise in obtained instantaneous velocity, we separated all the obtained data into a number of groups in each of which the data is in the same $1 \mathrm{~cm}$-square grid area and also in the time span of $0.1 \mathrm{~s}$. In each group, mean and standard deviation of the velocity components were estimated and the individual data whose deviation from the mean was 1.8 times greater than the standard deviation was removed as noise.

\section{Results and Discussions}

This section summarizes and analyzes experimental results of surface water fluctuations and horizontal velocity fields in front of the sea wall, respectively.

Characteristics of the Surface Water Fluctuations Figure 5 compares surface water fluctuations on the sea wall for both monochromatic and bichromatic incident wave cases, respectively. In the figure, the vertical axis is the measurement time while the horizontal axis corresponds to the locations along the seawall. In the figure, the darker color indicates the higher elevation of the instantaneous surface water level. While the figure shows only for the first $15 \mathrm{~s}$ of the measurements, the data was obtained for three minutes and all these data were used for computations of representative wave properties such as root-mean-square wave heights.

Since waves were obliquely incident on the basin, arrival time of the waves at the seawall has certain phase lag along the shore. Away from the vicinity of abruptly changing bottom slopes, i.e., in the area, $y<-100(\mathrm{~cm})$ or $100(\mathrm{~cm})<y$, for example, the observed crest lines in the Figure 5 are nearly straight but are sloping in the up-rightward direction. These up-rightward slopes indicate that the
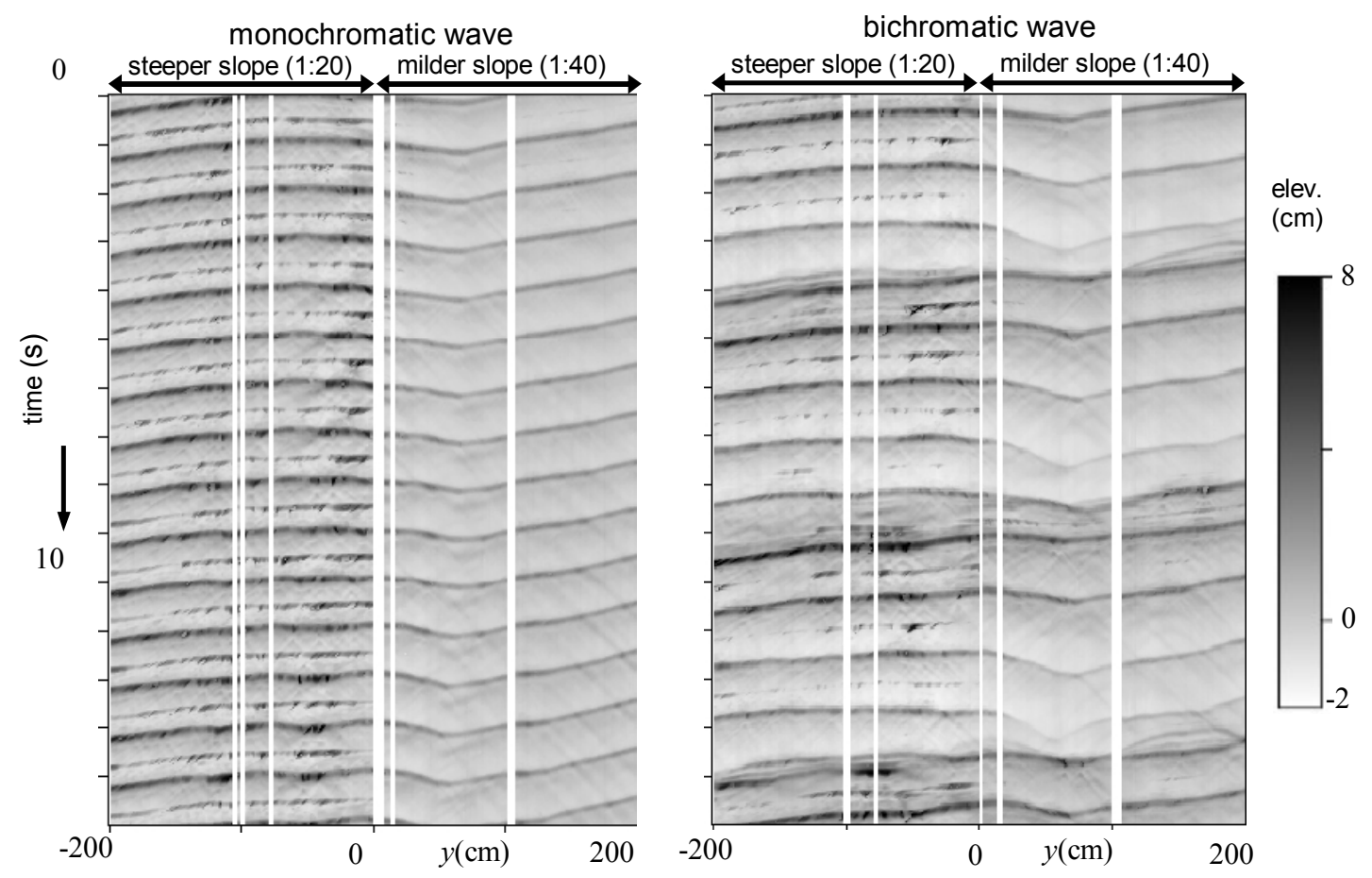

Figure 5 Surface water fluctuations at the seawall in both time and horizontal domains 
wave crest line arrived at the seawall earlier at the larger $y$ since the incident wave angle was from the right-offshore side of the basin. In the vicinity of the boundary of two different bottom slopes, i.e., in $100<y(\mathrm{~cm})<100$, wave refraction and diffraction curved the wave crest lines and, especially in the case of monochromatic wave, curving crest line profile has clear peak at around $y(\mathrm{~cm}) \sim 50$ on the milder 1:40 bottom slope. While the wave refraction should cause wave concentration around this peak, the observed wave heights did not show such clear evidence of the wave concentration in the case of monochromatic waves. Besides similar refraction and diffraction characteristics to the monochromatic waves, bichromatic incident wave case showed slowly varying envelopes with period of $5 \mathrm{~s}$. Alongshore locations of the time-peak of the curving wave crest line shown in Figure 5 also has slowly varying fluctuations with period of $5 \mathrm{~s}$.

In order to investigate the characteristics of slowly-varying long wave components, time-series of the surface water fluctuation at each alongshore location on the seawall was decomposed into discrete frequency components and, integrating these components whose frequency is either greater or smaller than $1 / 3 \mathrm{~Hz}$, the time series of either "long" or "short" components of surface water fluctuations were reproduced. Based on the obtained long and short components of surface water fluctuations, $H_{r m s}$, $H_{1 / 10}$, time-averaged water level, $\eta_{\text {mean }}$, was estimated. Figure 6 shows the alongshore distributions of $\eta_{\text {mean }}$ and wave heights of both short and long wave components for either monochromatic or bichromatic wave cases. As seen in the figure, there is clear difference between monochromatic and bichromatic wave cases and, especially in the case of bichromatic incident waves, $H_{\mathrm{rms}}$ of the long wave components, $H_{r m s, L}$ was significantly large. In the case of bichromatic incident wave, moreover, $H_{\text {rms }}$ and $H_{1 / 10}$ of the short wave components had local peak around $y \sim 100(\mathrm{~cm})$ on the milder 1:40 slope, while monochromatic incident wave case did not have such a local peak. The observed local peak of the wave heights on the milder slope is somehow consistent with the observed local peak of

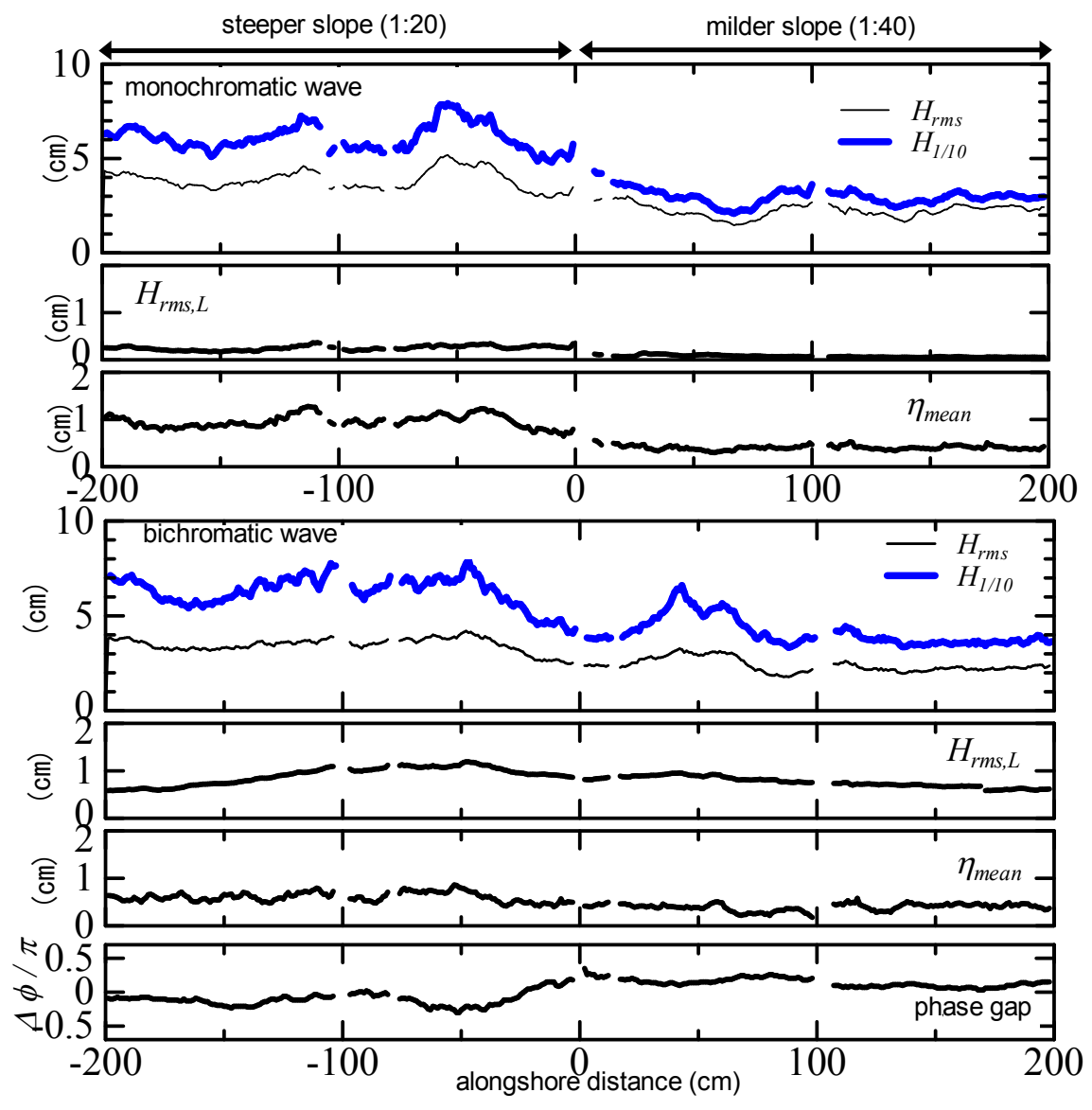

Figure 6 Alongshore distributions of measured wave heights, mean water levels and phase gaps. 
the inundation height on the milder slope near the abruptly changing bottom slopes behind the OisoSpur on the Seisho coast.

Surface Water Fluctuations on the Rectangular Light Panel Taking the same procedures as the surface water fluctuations on the seawall, surface water fluctuations on the rectangular light panel was also decomposed into each frequency components and, based on the reproduced fluctuations of long or short wave components, $H_{r m s}$ for both short and long wave components were estimated. Figure 7 compares horizontal distributions of the estimated $H_{r m s}$ of short and long wave components for monochromatic waves and bichromatic waves, respectively. Similar to the one observed in the surface water fluctuations on the seawall, clear difference between monochromatic and bichromatic waves were observed in terms of $H_{r m s}$ of long wave components. It is seen from the figure that $H_{r m s, L}$ has clear dominant undulations in the cross-shore direction and this feature somewhat indicates that long wave response may be dominant in the cross-shore direction.

In order to explore the interactive characteristics between low-frequency surface water fluctuations and slowly varying envelopes of the bichromatic waves, phase relationships of these waves were first investigated under the following procedures. Following List(1992), we first estimated the envelope function, $A(t)$, as a low-pass filtered profile of $|\eta(t)|$ with $\eta(t)$, time-varying surface water fluctuations at arbitrary location. Low-frequency component of the surface water fluctuations, $\eta_{L}(t)$, was also extracted using the same low-pass filter with maximum frequency of the low-pass filter set to be $0.5 \mathrm{~Hz}$. Obtained low-frequency surface water fluctuations, $\eta_{L}(t)$, was then separated into incident onshore-ward and reflected offshore-ward components, $\eta_{L, i}(t)$, and $\eta_{L, R}(t)$. Following Janssen and Battjes (2003), cross-correlation coefficient, $R(\tau)$, was then estimated by the following formulae,

$$
R(\tau)=\frac{1}{\sigma_{A 2} \sigma_{\eta_{L}}} \overline{A^{2}(t) \eta_{L, i}(t+\tau)}
$$

Here $\sigma_{A 2}$ and $\sigma_{\eta L, i}$ are standard deviations of $A^{2}(t)$ and $\eta_{L, i}$, respectively, and the overbar on the last two variables denotes the time-average over the entire time-series of the data. All these procedures were then applied to the time-series data of surface water fluctuations along the cross-shore line A shown in Figure 2 and also the alongshore line on the seawall.

Figure 8 shows the distributions of computed cross-correlation coefficients, $R(\tau)$, as function of time shift, $\tau$, and horizontal distance either along the line A or along the seawall, respectively. Horizontal axis of the figure denotes the location either along the line A or along the seawall and the vertical axis denotes the phase shift, $\Delta \phi=2 \pi \tau / T_{L}$, defined as a function of the time shift, $\tau$, and $T_{L}=5 \mathrm{~s}$,

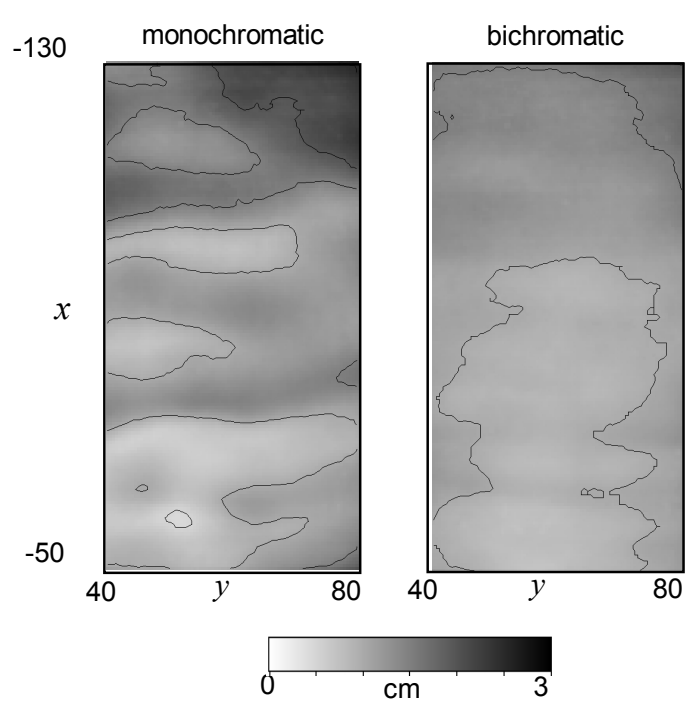

(a) short wave components $(T<3 s)$

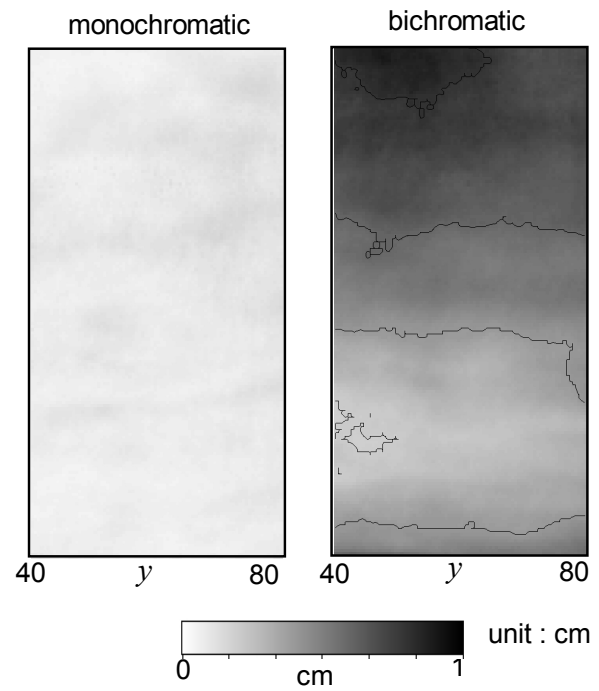

(b) long wave components $(T>3 s)$

Figure 7 Horizontal distributions of $\boldsymbol{H}_{\mathrm{rms}}$ of (a) short and (b) long wave components on the light panel 


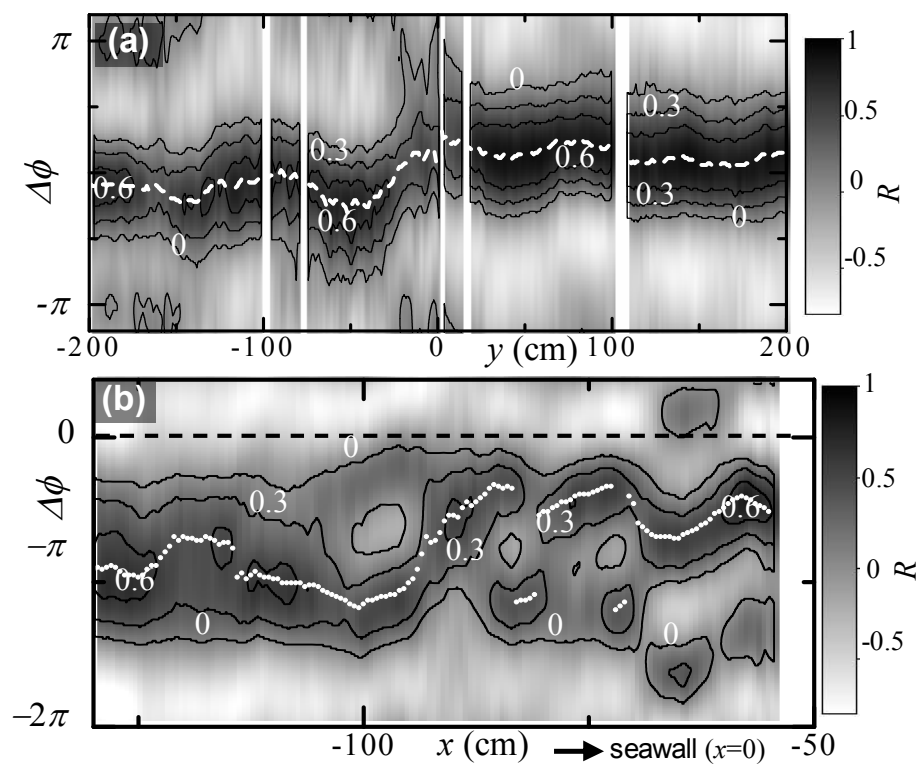

Figure 8 Alongshore $(y)$ and cross-shore $(x)$ distributions of cross-correlation coefficient, $R$, as a function of the phase gap, $\Delta \phi$, of wave group envelopes and long waves

the period of slowly varying wave envelope. White dashed line in the figure indicates the phase shift at which the cross-correlation coefficient reached its peak, i.e., profiles of envelope and low-frequency surface water fluctuation best matches with each other.

As seen in the figure, phase shift decreases as the wave propagate toward the shore along the line A and this feature surely corresponds to the similar experimental results (e.g., Janssen and Battjes, 2003, and Seki and Mizuguchi, 2008). On the seawall, on the other hand, the phase of envelope and the low-frequency surface water fluctuations match with each other. This feature suggests that the peak of the wave heights in the envelope comes with the peak of the surface water fluctuations. Since the higher water level yields larger water depth and the larger water depth should decrease the broken wave dissipation rate, i.e., increase the broken wave heights, observed match of the phases between envelope and slowly varying surface water fluctuations may yield larger wave height in front of the seawall. This feature is further investigated in the following numerical experiments.

Current Velocity Fields Figure 9 shows the time-averaged horizontal current velocity fields for both monochromatic and bichromatic wave cases estimated from succeeding images recorded by the high-speed camera. The figure also shows the cross-shore distributions of the horizontal velocity components in the alongshore and the cross-shore directions, respectively. These representative velocity components were obtained by averaging local velocity components along the respective shoreparallel line. The figure also shows the cross-shore distributions of predicted depth-averaged horizontal velocity components in the alongshore and cross-shore directions, which will be discussed later in the next section of the numerical experiments. As seen in the figure, the time-averaged velocity fields in the measured area were nearly uniform in both monochromatic and bichromatic wave cases but magnitude of the current velocity was about two times greater in the case of bichromatic waves than monochromatic waves while the time-averaged incident wave energy and incident wave angles of theses waves are equivalent to each other. This feature is reasonably represented by the numerical model in predictions of the depth-averaged longshore currents.

\section{NUMERICAL ANALYSIS}

Laboratory experiments successfully represented the locally concentrating wave field in the vicinity of abruptly changing bottom slopes near the shore. Clear difference between monochromatic waves and bichromatic waves with slowly varying wave envelopes was also observed especially in that wave concentration on the milder slope appeared only in the case of bichromatic waves. This section aims to further investigate the physical mechanisms of such wave concentrations observed through the 


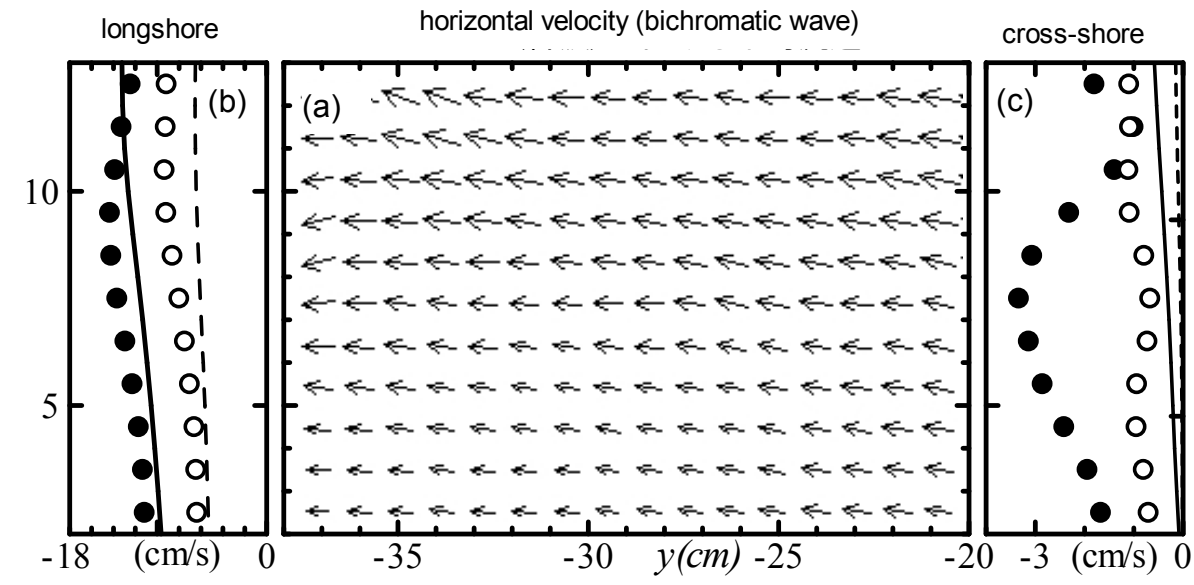

Figure 9 Horizontal distributions of the time-averaged current velocity under bichromatic waves (a) and longshore (b) and cross-shore(c) components of horizontal velocity both under monochromatic and bichromatic waves (solid and blank circles; measured data and solid and dashed lines; predictions respectively for monochromatic and bichromatic waves)

laboratory experiments. Special focus is put on comparisons of relative importance of possible factors that could cause such wave concentration near the shore.

In order to explore the hydrodynamic difference between monochromatic and bichromatic incident wave conditions, the numerical model should be able to represent the propagations of slowlyvarying wave envelopes and resulting slowly fluctuating wave radiation stress that induces lowfrequency waves as well as the near shore currents. Interactions among short waves, long waves, and nearshore mean current should also be accounted for in the model.

\section{Model Descriptions}

While time-dependent non-linear dispersive model such as Boussinesq-type model can directly solve such interactive features of waves and currents, computed horizontal current velocities and surface water fluctuations contain all of these hydrodynamic properties and it is therefore virtually difficult to separate those into each component to investigate their direct impact on the wave deformations. In this study, therefore, we applied time-dependent mild slope equations based on linear wave theory for computations of the short wave components and time-dependent non-linear shallow water equations for the computations of nearsore currents as well as slowly varying low-frequency waves. These two models were iterated so that the interactive features among short wave, long wave and time-averaged nearshore curernts and wave setups are reasonably accounted for.

This study applied the following Oonaka and Watanabe's (1987) time-dependent mild slope equations for computations of short wave propagations,

$$
\begin{gathered}
\left(1+\frac{\sigma}{\omega}\left(\frac{C_{g}}{C}-1\right)\right) \frac{\partial \zeta}{\partial t}+\nabla \cdot(\mathbf{U} \zeta)+\nabla \cdot\left(\frac{C_{g}}{C} \mathbf{Q}\right)=0 \\
\frac{\partial \mathbf{Q}}{\partial t}+\omega C^{2} \nabla \cdot\left(\frac{\zeta}{\sigma}\right)=-D_{b}
\end{gathered}
$$

where $\omega$ and $\sigma$ are the wave angular frequencies respectively on fixed coordinate system and on moving coordinate systems with current speed, U. Momentum equation, (5), moreover, introduces breaking and broken wave dissipation term, $D_{b}$, proposed by Tajima et al. (2007) which is verified to be applicable to relatively complex arbitrary wave fields including partially reflected waves. As seen in these momentum and continuity equations, the time-dependent mild slope equations first need to specify the dispersion relationships of the propagating waves, i.e., to determine both wave phase velocity and group velocity on all the computation domain. Since the wave dispersion relationship is determined as a function of local water depth and a wave period, the model requires special treatments for computations of waves with multiple frequency components such as bichromatic waves. For computations of bichromatic waves, this study simultaneously solved two separate sets of momentum and continuity equations for each wave frequency components and linearly integrated these results to 
obtain instantaneous surface water fluctuations and wave orbital velocity fields. These combined instantaneous properties of bichromatic waves were then used to determine time-varying non-linear breaking and broken wave dissipation term, $D_{b}$, which should be highly affected by combinations of these two wave components.

For computations of slowly varying long wave including time-averaged nearshore current and wave setups, this study simply applied non-linear shallow water equations with additional terms of bottom shear stress, mixing dispersions, and wave radiation stress. Madsen's (1994) bottom boundary layer model was used to evaluate bottom shear stress due to nearshore current under wave-current interactive field and spatially varying dispersion coefficients for nearshore current fields were determined by Larson and Kraus's (1991) model. In order to account for the time-varying waveinduced forces due to slowly varying wave envelopes, this study computed the wave radiation stress through the following numerical procedures. Following Longuet-Higgins and Stewart (1962), the model first computed "instantaneous wave radiation stress," $R{ }_{i}^{\prime}$, defined by

$$
R_{i}^{\prime}=\frac{\partial S_{j i}^{\prime}}{\partial x_{j}}
$$

in the computations of short wave field based on the time-dependent mild slope equations. Here subscripts $i$ and $j(=1,2)$ denote the components in the horizontal $x$ and $y$ directions and

$$
S_{i j}^{\prime}=\frac{\rho}{2} \zeta^{2} \delta_{i j}+\int_{-h}^{0} \rho\left(u_{i} u_{j}-w^{2} \delta_{i j}\right) d z
$$

is the instantaneous radiation stress tensor with $\zeta$, instantaneous surface water levels of combined waves, $\delta_{i j}$, Kronecker's delta, $u_{i}$, wave-associated horizontal velocity components in the $i$-th direction, and $w$, wave-associated vertical velocity component. In the case of monochromatic waves, monochromatically changing $R_{i}^{\prime}$ were averaged over a wave period to determine the time-averaged Radiation stress and the estimated constant Radiation stress was then simply introduced in the nonlinear shallow water equations for computations of time-averaged nearshore current. In the case of bichromatic waves, on the other hand, slowly-varying radiation stress was first computed by extracting the low-frequency components of $R_{i}^{\prime}$. Obtained $R_{i}^{\prime}$ were then introduced in the non-linear shallow water equations by changing their values as the time proceeds with the actual numerical computations.

\section{Experimental Cases and Conditions}

Based on the numerical models described in the previous section, this study performed the numerical experiments. In the experiments, wave fields based on the mild slope equations and the nearshore current fields including the slowly varying components based on the non-linear shollow water equations were iterated so that each model can feed back their computed results to the other. During these iterations, this study neglected a part of hydrodynamic features as indicated by " $x$ " in Table-1 for different computation cases. Relative importance of various hydrodynamic factors can therefore be observed through comparisons of experimental cases in Table-1.

Table 1 Cases of numerical experiments

\begin{tabular}{|c|c|c|c|c|c|}
\hline \multirow{2}{*}{ cases } & wave & \multicolumn{2}{|c|}{ time-averaged components } & \multicolumn{2}{|c|}{ long wave components } \\
\cline { 3 - 6 } & breaking & water level & current & water level & current \\
\hline A & $\mathbf{X}$ & $\mathbf{X}$ & $\mathbf{X}$ & $\mathbf{X}$ & $\mathbf{X}$ \\
\hline B & $\circ$ & $\mathbf{X}$ & $\mathbf{X}$ & $\mathbf{X}$ & $\mathbf{X}$ \\
\hline C & $\circ$ & $\circ$ & $\mathbf{X}$ & $\mathbf{X}$ & $\mathbf{X}$ \\
\hline D & $\circ$ & $\circ$ & $\circ$ & $\mathbf{X}$ & $\mathbf{X}$ \\
\hline E & $\circ$ & $\circ$ & $\circ$ & 0 & $\mathbf{X}$ \\
\hline F & $\circ$ & $\circ$ & 0 & 0 & 0 \\
\hline
\end{tabular}

The other computation conditions were set so as to represent the present laboratory experiments. As shown in Figure 2, the computation was performed on a rectangular area with cross-shore and alongshore distances of 4 and 8 meters, respectively. Incident waves were introduced uniformly along the straight offshore boundary and perfect reflection boundary conditions were applied on both alongshore sides of the basin to represent the vertical wall boundaries of the basin. In order to absorb the short wave components, 30 -cm-wide sponge layers were installed on both sides of the basin and to 
represent this feature, the model applied artificial dissipation term which is proportional to local instantaneous current velocity. Along the on shore seawall, perfect reflection condition was also applied.

\section{Numerical Results}

Figure 10 shows computed wave and current fields when either monochromatic or bichromatic waves were obliquely incident on the basin. In the figure, horizontal distributions of the root-meansquare wave heights are expressed in gradually changing color and contour lines whereas the horizontal current velocity is represented in vectors. It is clearly seen in the figure that the wave tends to concentrate where the seaward current velocity component is dominant in both monochromatic and bichromatic wave cases and the different current patterns somehow appear to determine the horizontal locations of the wave concentrations. It is also seen in the figure that computed nearshore current velocity under bichromatic waves is uniformly distributed on the steeper slope near the abruptly changing bottom slopes as was observed in the experimental results shown in Figure 9. Figure 9 also compares the predicted and measured cross-shore distributions of the current velocity components averaged over the shore-parallel lines. As seen in this comparison, the predicted longshore current velocity agree well with the measured data even in a quantitative manner in both monochromatic (solid line) and bichromatic (dashed line) incident wave cases. It should be noted that the model underestimated the seaward current velocity components since the model only predicts depth-averaged

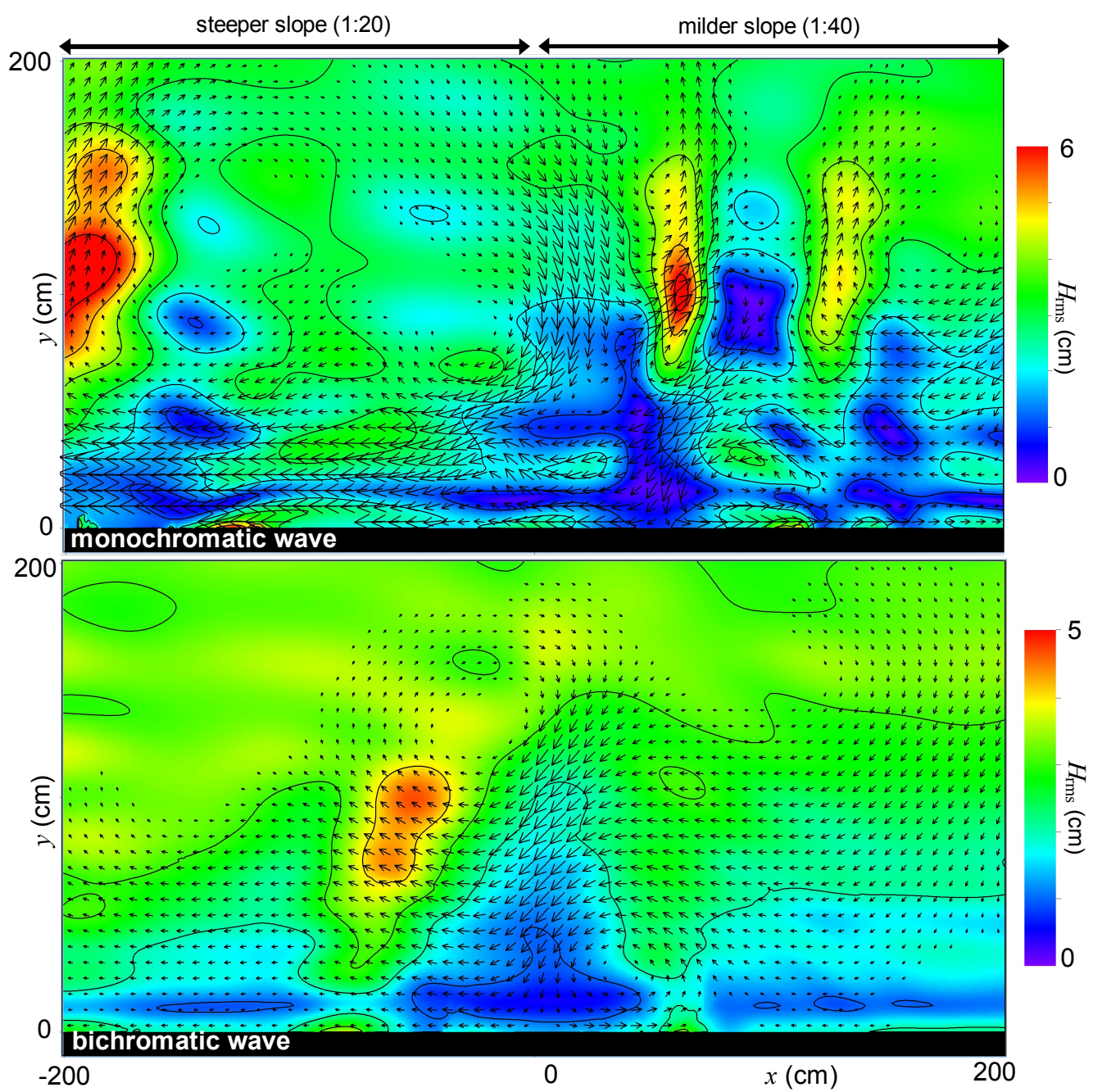

Figure 10 Predicted root-mean-square wave heights and time-averaged nearshore current velocity for both monochromatic wave (case D) and bichromatic wave (case F). 
current velocity which does not account for the influence of the undertow. On the other hand, the observed velocity is the near-bottom velocity components measured at $5 \mathrm{~mm}$ above the bed and should be affected by the undertow.

Finally, Figures 11 compare the alongshore distributions of the predicted and measured wave heights along the seawall. The figure compares computed results based on different cases outlined in Table 1. Figure 11(a), for example, compares the wave heights along the seawall when the model only accounted for wave deformations due to shoaling, refraction and diffraction, i.e., the model neglected various deformation factors such as breaking and broken wave dissipations and interactions among short waves, long waves and time-averaged nearshore currents. As seen in the figure, both monochromatic and bichromatic waves had a local peak on the milder 1:40 slope near the boundary where the bottom slope changes while observed distributions of monochromatic waves had no such local peak on the milder slope. Moreover, the predicted peaks were milder than the measured one and, compared to the measured data, the alongshore location of the predicted peak was closer to the center of the bathymetry, i.e., the boundary between steeper and milder bottom slopes. Based on these observations, it is clear that the simple wave refraction phenomena of the short wave components do not reasonably explain the nearshore wave concentrations. It is also interesting to note that the predicted wave heights were slightly larger on the milder slope while the measured wave heights tended to be larger on the steeper slope both in monochromatic and bichromatic wave cases. This feature indicates the importance of the broken wave dissipation terms as functions of different bottom

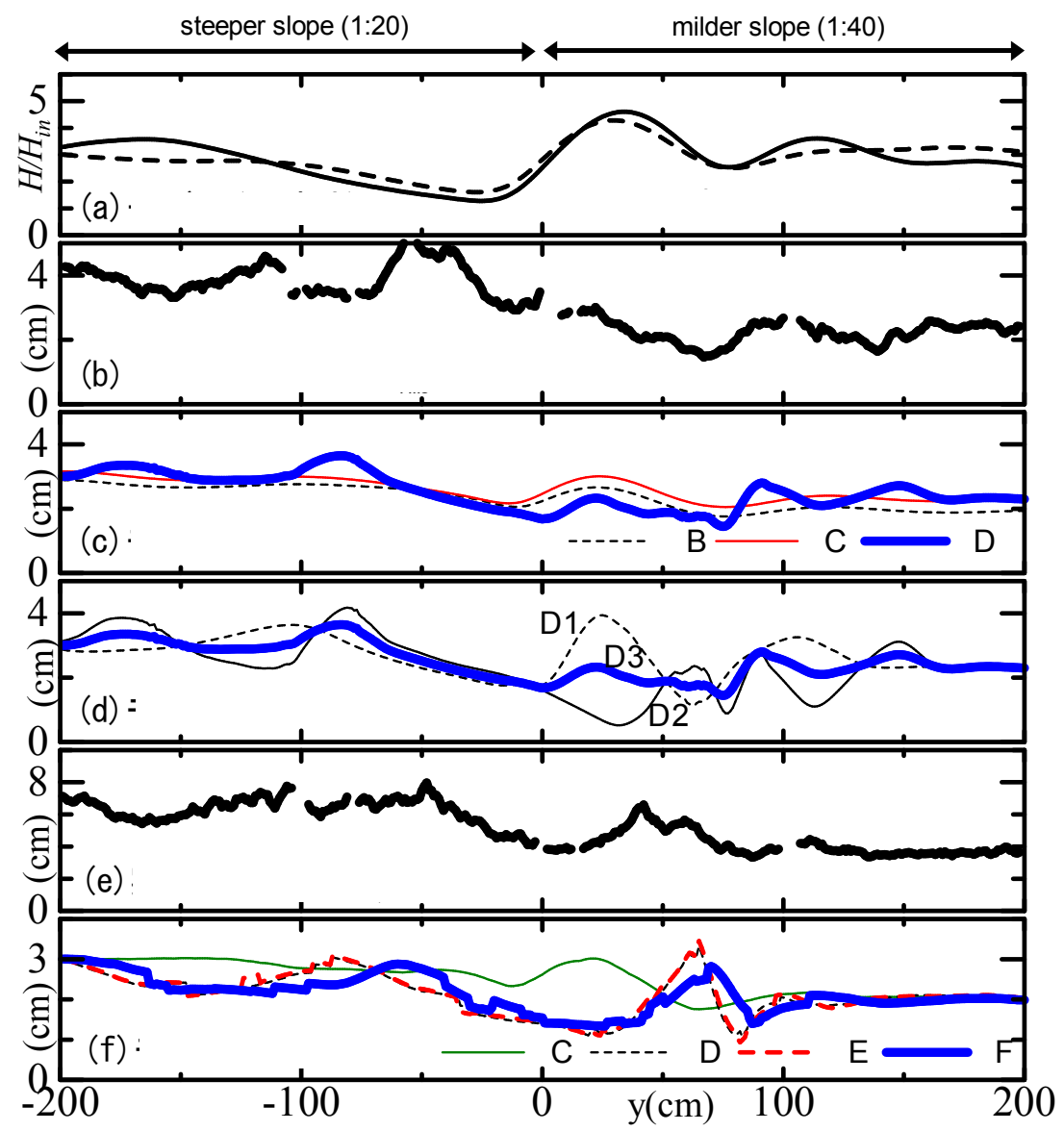

Figure 11 Comparisons of predicted and measured wave heights along the seawall for monochromatic wave (a, b, c, d) and bichromatic wave (a, e and f). (a) predicted non-breaking (case A) monochromatic (solid line) and bichromatic (dashed line) waves, (b) measured $H_{\mathrm{rms}}$, (c) predicted cases B, C and D, (d) case $D$ with different iteration number for computations of wave-current interactions, (e) measured $H_{1 / 10}$, and (f) predicted cases C, D, E, and F. 
slopes.

Figure 11(c) compares cases B, C and D when monochromatic waves were incident on the basin. As outlined in Table 1, the cases B, C and D added the following wave-deformation factors one by one: (i) wave breaking; (ii) increase of the water depth due to wave setup; and (iii) wave-current interactions due to nearshore mean current. As seen in the case $C$, breaking wave model successfully captured the feature that the wave heights on the steeped slope tended to be larger than those on the milder slope except around the local peak. Difference between $\mathrm{C}$ and D, i.e., the influence of mean current on wave deformation, is more significant compared to the difference between $\mathrm{B}$ and $\mathrm{C}$, i.e., the influence due to wave setup. By introducing the interactions between nearshore mean current and short waves, as seen in the case D, predictive skills of the alongshore distributions of the wave heights are largely improved especially in that the local peak on the milder slope at around $\mathrm{y}=40 \mathrm{~cm}$ observed in computed cases $\mathrm{A}, \mathrm{B}$ and $\mathrm{C}$, were diminished and instead a local peak was developed on the steeper bottom slope at around $y=-70(\mathrm{~cm})$ while the observed peak, located around $\mathrm{y}=-50(\mathrm{~cm})$, was slightly offset.

Figure 11 (d) compares the wave heights in case D but when the number of iteration of wave and current computations was altered. In the figure, the numbers in D1, D2, and D3 denote how many times the wave-current interactions were iteratively computed. In the case of D1, for example, wave field was computed twice so that the second wave computation accounts for the influence of the nearshore current induced by the wave radiation stress based on the first wave computation. As shown in the figure, the computed results changed especially in the vicinity of the boundary of milder and steeper bottom slopes and the best predictive skills was observed in the case of D3. It was also confirmed that the computed results nearly converged after three-time iterations.

Figures 11 (e) and (f) compare the measured wave heights and the predicted ones in cases, C, D, $\mathrm{E}$ and $\mathrm{F}$, respectively, when the bichromatic waves were incident on the basin. In order to investigate the influence of slowly varying components of the surface water fluctuations or current velocity, we applied the same computed results of the slowly-varying nearshore current field for computations of wave-current interacting wave fields but extracted either fluctuating or time-averaged components of computed results depending on the cases outlined in Table 1. The case E, for instance, directly substituted computed $\mathbf{U}$, which contains both time-averaged and slowly fluctuating components, into the mild slope equations, (4) and (5), whereas the case E applied only the time-averaged components of the surface water level, $\eta_{\text {mean }}$, for computations of the instantaneous local water depth used in the equations (4) and (5). In Figures (e) and (f), alongshore distributions of $H_{1 / 10}$ were compared in stead of $H_{\mathrm{rms}}$ since this study focus more on the concentrations of larger waves that directly cause severe coastal damages. As seen in the Figure (f), the model tends to underestimate the wave heights along the seawall while the model successfully captured the alongshore profiles of wave heights, i.e., locally concentrating features of the waves. This underestimation may be because the model is based on the linear wave theory and thus could not capture the sharp crest of the surface water profile in front of the seawall. Comparison of cases C, D, E, and F reveals that the influence of the slowly-varying water depth, represented by the difference between cases D and E, is less significant compared to the influence of interactions between waves and time-averaged mean current, represented by a difference between cases C and D. Since, as seen in Figure 8, the phases of wave envelope and slowly-varying surface water fluctuations match with each other, increase of the instantaneous water depth due to low-frequency surface water fluctuations may decrease the broken wave dissipation rate and thus increase the broken wave height. In this numerical experiment, the influence of such surface water fluctuations was not as significant as the influence of wave-current interactions. Slowly-varying component of the nearshore current, as seen in comparisons between $\mathrm{E}$ and $\mathrm{F}$, affect to change the alongshore locations of the local peaks of the wave heights while its impact was less significant than that of time-averaged components of the nearshore current.

\section{CONCLUSIONS}

Laboratory experiments were performed to investigate the physical mechanisms of locally concentrating wave fields around the abruptly changing bottom slopes. Besides simple monochromatic waves, this study applied bichromatic waves with slowly varying wave envelopes, which induced the generation of low-frequency wave components. Image-based measuring system was newly developed and applied to the experiments. This measuring system enabled us to capture the wave and current fields in wider area with higher spatial and time resolutions. The experiments with this measuring 
system successfully captured such wave concentration phenomena especially around the abruptly changing bottom slopes.

Through the experiments, clear difference between monochromatic waves and bichromatic waves were observed while their time-averaged wave energy and the mean wave periods were equivalent with each other. While the monochromatic wave had no local peak of the wave height on the milder slope, bichromatic wave case had a clear local peak of the wave height near the alongshore boundary of the two different bottom slopes.

Numerical experiment was then performed to further investigate the detailed physical mechanisms of the observed wave concentrations. In order to decompose various factors of wavedeformations which affect wave concentrations, this study applied linear time-dependent mild slope equations for computations of short wave fields and non-linear shallow water equations with timevarying wave radiation stress for computations of nearshore current and slowly-varying long wave components. These two models were iteratively applied so that the entire model can accommodate interactions among short waves, long waves and time-averaged nearshore currents. During the iteration, different numerical cases selected a different part of various interactive factors so that comparisons of computed results in these different cases represent the influence of some specific factors.

Accounting for all the interactive features of short waves, long waves and time-averaged nearshore currents, the present model reasonably explained the difference between monochromatic and bichromatic incident wave particularly in that the local peak of the wave height on the milder slope was observed only in the case of bichromatic incident waves. Through the numerical experiments, it was also found that, among various interactive factors, interactions between timeaveraged nearshore current and the short wave components were one of the most dominant factors that determine the nearshore wave fields around the abruptly changing bottom slopes.

\section{ACKNOWLEDGMENTS}

Authors would like to acknowledge with sincere gratitude that this research was supported by a grant-in-aid of JRTT, Japan Railway Construction, Transport and Technology Agency.

\section{REFERENCES}

Janssen, T.T., J.A. Battjes, and A.R. van Dongeren. 2003. Long waves induced by short-wave groups over a sloping bottom, J. Geophy. Res. 108, C8, 3252, doi:10.1029/2002JC001515.

Larson, M. and N.C. Kraus. 1991. Numerical model of longshore current for bar and trough beaches, J. Waterway, Port, Coast. and Ocea. Eng., vol.117, No.4, 326-347.

List, J.H. 1992. Wave groupiness variation in the nearshore, Coast. Eng., vol.15, 475-496.

Longuet-Higgins, M.S. and R.W. Stewart. 1962. Radiation stress and mass transport in gravity waves, with application to "surf beat," J. of Fluid Mechanics, vol.13, 481-504.

Madsen, O. S. 1994. Spectral wave-current bottom boundary layer flows, Proc. 24th Int. Conf. on Coast. Eng., 384-398.

Ohnaka, S., A. Watanabe and M. Isobe. 1989. Numerical computation of wave field by the unsteady mild slope equation extended to wave-current coexisting field, Proc. of Coast. Eng., JSCE, 91-95 (in Japanese).

Seki, K., and Y. Mizuguchi. 2008. Shoaling of long period waves induced by grouping short-waves on sloping bottom. J. of the Japan Society of Civil Engineers B, JSCE, vol.64, 62-70 (in Japanese).

Tajima, Y., H. Liu and S. Sato : Dynamic changes of waves and currents over the collapsing sandbar of the Tenryu river mouth observed during Typhoon T0704, Proc. Coastal Dynamics 2009, CDROM published by World Scientific.

Tajima, Y., S. Sato, T. Shimozono and M. Isobe. 2007. Characteristics and modeling of wave-induced current around submerged detached breakwaters, Proc. Int. Conf. on Coastal Structures, 725-736.

Tajima, Y. and S. Sato. 2009. Numerical investigations of locally concentrated damages on Seisho coast sue to typhoon T0709, Proc. of 5th Int. Conf. on Asian and Pacific Coasts. 